\title{
La prose littéraire des Grecs d'Australie : enjeux identitaires
}

\section{Stéphane Sawas}

\section{(2) OpenEdition \\ 12 Journals}

Édition électronique

URL : http://journals.openedition.org/jso/5952

DOI : $10.4000 /$ jso. 5952

ISSN : 1760-7256

Éditeur

Société des océanistes

\section{Édition imprimée}

Date de publication : 15 décembre 2009

Pagination : 241-247

ISBN : 978-2-85430-026-0

ISSN : 0300-953x

\section{Référence électronique}

Stéphane Sawas, "La prose littéraire des Grecs d'Australie : enjeux identitaires », Journal de la Société des Océanistes [En ligne], 129 | juillet-décembre 2009, mis en ligne le 30 décembre 2012, consulté le 19 avril 2019. URL : http://journals.openedition.org/jso/5952 ; DOI : 10.4000/jso.5952

(c) Tous droits réservés 


\section{La prose littéraire des Grecs d'Australie : enjeux identitaires}

par

\section{Stéphane SAWAS*}

\section{RÉSUMÉ}

Représentatifs des principaux types d'immigration grecque aux antipodes, les écrivains V. Kalamara, A. Kefala et Ch. Tsiolkas sont liés à la Grèce et à l'Australie de manière différente. D'une écriture de l'exil à la revendication d'une culture wog, leurs démarches mettent au jour l'évolution des problématiques identitaires dans l'Australie de la fin $d u x^{e}$ siècle et du début $d u$ $X_{X I}^{e}$ siècle, qui a un impact tant sur la thématique et l'esthétique que sur la diffusion et la réception de leurs auvres.

MoTS-CLÉS : littérature, identité, immigration grecque, multiculturalisme australien, exil, diaspora, wog

La littérature des Grecs d'Australie fait depuis près de vingt ans l'objet de recherches qui s'inscrivent dans le prolongement des débats, parfois houleux et contradictoires, sur le multiculturalisme australien (Wapshere, 1994 ; Pons, 1996). L'ouvrage pionnier du philologue Yorgos Kanarakis, La Présence littéraire des Grecs en Australie, publié en grec en 1985 et en anglais en 1987 (Kanarakis, 1985 et 1987), propose une approche historique et une anthologie de textes souvent parus à compte d'auteur ou dans des revues confidentielles, tant en grec qu'en anglais, qui montrent l'importance quantitative, méconnue jusqu'en Grèce, de ce vaste corpus littéraire. À la suite de ce travail à bien des égards fondateur, les études menées sur ces œuvres sont souvent subordonnées aux débats sur le multiculturalisme. Or, les considérations sur le multiculturalisme littéraire en Australie, tout comme celles relatives au concept d'écriture migrante au

\section{ABSTRACT}

The writers V. Kalamara, A. Kefala and Ch. Tsiolkas, bound up with Greece and Australia in different ways, represent the main patterns of Greek immigration to the Antipodes. From writing about exile to claiming a wog culture, they highlight the evolution of cultural and ethnic identity in Australia at the end of the $20^{\text {th }}$ and the beginning of the $21^{\text {st }}$ century. This evolution both influences their themes and aesthetics as well as the way their work is read and received.

KEYWORDS: literature, identity, Greek immigration, Australian multiculturalism, exile, diaspora, wog

Canada (Harel, 2005), interrogent davantage le canon littéraire et, plus largement, l'identité du pays d'accueil, que les œuvres regroupées dans un corpus qualifié de « migrant » ou de « multiculturel », constitué de textes situés précisément aux confins de canons littéraires nationaux (Sawas, 2007 et 2008).

L'œuvre des prosateurs Vasso Kalamara, Antigone Kefala et Christos Tsiolkas permet de mettre au jour la diversité des contributions des écrivains grecs d'Australie, trop souvent réunies hâtivement dans une même catégorie. Représentatifs des principaux types d'immigration grecque aux antipodes, ces auteurs, à la fois grecs et australiens, liés toutefois à la Grèce et à l'Australie de manière très différente, portent des regards nécessairement éloignés sur l'identité des Grecs d'Australie, ce qui n'est pas sans incidence sur la diffusion et la réception de leur œuvre tant en Australie qu'en Grèce.

* Maître de conférences, INALCo-Paris, stephane.sawas@gmail.com 


\section{De la Grèce à l'Australie}

En abordant ce que l'on appelle littérature des Grecs d'Australie, littérature gréco-australienne ou, plus prudemment, présence littéraire des Grecs en Australie, on a souvent tendance à sous-évaluer l'hétérogénéité du corpus étudié. Or, l'œuvre de Vasso Kalamara, d'Antigone Kefala et de Christos Tsiolkas conduit en premier lieu à remarquer que la littérature des Grecs d'Australie est placée sous le signe de la diversité. En effet, si ces trois écrivains sont de parents grecs et vivent en Australie, ils n'en entretiennent pas moins des liens à la Grèce et à l'Australie très différents qui conditionnent leur approche de l'identité gréco-australienne.

Remarquons d'abord qu'ils représentent deux générations d'auteurs grecs d'Australie : Vasso Kalamara, née au début des années $1930^{1}$, arrive en Australie en 1951 et publie son premier livre en 1959; Antigone Kefala, née en 1935, arrive en Australie en 1959 et publie son premier livre en 1973 ; Christos Tsiolkas naît en Australie en 1965 et publie son premier livre en 1995.

La répartition de ces auteurs sur le territoire australien mérite ensuite quelques commentaires : Vasso Kalamara vit à Perth et revendique son indépendance à l'égard de la côte Est, où se trouvent les principaux foyers de la communauté grecque d'Australie et les centres culturels majeurs du pays ; de façon plus attendue, Antigone Kefala vit à Sydney et Christos Tsiolkas à Melbourne.

Ces écrivains ne sont en outre guère représentatifs de l'image dominante des immigrés contraints à quitter la Grèce pour des raisons économiques. En effet, Vasso Kalamara, Antigone Kefala et les parents de Christos Tsiolkas viennent de Grèce et arrivent en Australie dans des conditions très différentes.

Vasso Kalamara, d'origine crétoise et rouméliote, naît à Athènes, y passe son enfance et son adolescence dans une famille aisée, y est scolarisée en grec et initiée aux arts. Elle rencontre à l'École des Beaux-Arts son mari, sculpteur originaire de Macédoine, et quitte la Grèce en 1951 pour rejoindre son époux et son beau-père, lequel s'est installé dans les années 1920 en Australie occidentale. Le couple part dans l'idée de trouver un climat favorable à leur art respectif, mais les travaux à la ferme du beaupère, située à 300 kilomètres au sud de Perth, sont peu propices à la création artistique et isolent les Kalamaras dans un îlot hellénophone, coupé du monde anglophone. Vasso Kalamara écrit, peint, sculpte et, à partir de la fin des années 1960, enseigne le grec moderne à des étudiants australiens à Perth. Ses poèmes, nouvelles, pièces de théâtre et récit de voyage sont, à une exception près, écrits en grec.

Le cas d'Antigone Kefala est encore plus complexe. Elle naît en 1935 en Roumanie, à Braïla, de parents grecs, et gagne avec sa famille la Grèce après la Seconde Guerre mondiale en qualité de réfugiée. Alors qu'en Roumanie, elle parlait principalement le français et le roumain, elle apprend en Grèce le grec qu'elle connaissait mal. Les parents d'Antigone Kefala préfèrent toutefois quitter la Grèce de la guerre civile, qui réserve un accueil mitigé à cette famille de la prospère diaspora grecque de Roumanie, qui a tout perdu, et émigrent en Nouvelle-Zélande en 1951. Antigone Kefala y apprend l'anglais, étudie la littérature française à l'Université de Wellington et commence à écrire en anglais, sa quatrième langue ${ }^{2}$. Elle s'installe, pour des raisons personnelles, à Sydney en 1959, à la fin de ses études, et publie en Australie poèmes et nouvelles en anglais.

Enfin, Christos Tsiolkas, souvent présenté comme une personnalité sulfureuse en marge de la communauté grecque, est pourtant issu d'une famille plus en accord avec l'image dominante que l'on se fait des immigrés grecs en Australie. Ses parents quittent la Grèce pour des raisons économiques; il naît à Melbourne dans un quartier populaire peuplé d'immigrés principalement originaires d'Europe du Sud et de Méditerranée orientale. Il est scolarisé en anglais en Australie et pratique le grec surtout dans le cercle familial. C'est donc naturellement en anglais qu'il écrit ses romans, pièces de théâtre et scénarii.

Ainsi, ces trois auteurs, gréco-australiens suite à des choix esthétiques, à des nécessités politiques ou à des contingences économiques, représentent trois des principaux types d'immigration grecque vers les antipodes : immigration d'élection dans un contexte post-colonial avec Vasso Kalamara, immigration de réfugiés politiques en provenance des anciens foyers de la diaspora avec Antigone Kefala, immigration économique d'après-guerre avec Christos Tsiolkas. Ils portent dans leur œuvre un regard nécessairement différent sur l'identité gréco-australienne.

1. L'écrivain ne mentionne à notre connaissance pas sa date de naissance. Nous suivons donc sur ce point la présentation biographique de Marie Gaulis (Gaulis, 2001 : 125).

2. $C f$. l'entretien qu'accorde Antigone Kefala à Helen Nickas (Nickas, $1992: 225 s q$ ). 


\section{Émigrés, immigrés et wogs}

La représentation de l'identité gréco-australienne, de Vasso Kalamara à Christos Tsiolkas, évolue selon une ligne de partage qui n'est pas réductible à l'opposition entre une première et une seconde génération de Grecs d'Australie.

Vasso Kalamara qualifie au moins à deux reprises ses textes de gréco-australiens : sur la page de garde, les poèmes de Paysage et âme (Topio kai psychi, 1980) sont présentés comme " poèmes gréco-australiens » (« ellinoafstraliana piimata ») (Kalamaras, 1980b) et les nouvelles de Autres terres (Alla chomata, 1961), du moins dans les rééditions de 1977 à Perth et de 1980 à Athènes, comme "nouvelles grécoaustraliennes » (《Greek-Australian stories 》 ou « ellinoafstraliana diiyimata ») (Kalamara, 1977 et 1980a). Ils ne rendent pourtant guère compte des spécificités identitaires des Grecs d'Australie.

Les nouvelles du recueil Autres terres sont par exemple principalement situées en milieu rural alors que la grande majorité des immigrés grecs vivent en milieu urbain. Les personnages principaux, souvent des mères de famille, sont presque toujours des immigrés de première génération qui ne vivent que dans le souvenir de leur vie passée en Grèce, sans espoir de retour. Les enfants n'ont en outre guère la possibilité de construire une vie et d'affirmer une identité dans le pays d'accueil qu'est l'Australie. Citons par exemple le personnage du fils dans la nouvelle « Alkis, ou Allan, le petit Grec » (« Alkis, i Allan, o mikros Graikos »), qui ouvre le premier recueil de nouvelles de l'auteur, Autres terres : attiré par le bush australien, le garçon s'y perd et y est retrouvé mort pendant que la mère prie la Vierge et implore sa propre mère (c'est-à-dire la grandmère du petit), restée en Grèce.

Le genre littéraire principal dont on perçoit l'influence à la lecture tant des poèmes que des nouvelles de Vasso Kalamara est la chanson dite de l'exil (tis xenitias), genre à part de la chanson traditionnelle grecque (Gaulis, 2001 : 144-146). L'auteur s'inscrit volontairement dans un univers littéraire grec et populaire, comme le montre également son théâtre: ses pièces Karaghiozis riche (O Karaghiozis ploussios, 1987), Karaghiozis milkbariste (O Karaghiozis milkbaristas, 1987), Karaghiozis interprète (O Karaghiozis diermineas, 1987), Karaghiozis immigré (O Karaghiozis metanastis, 1992), mettent en scène en Australie le personnage du théâtre d'ombres grec Karaghiozis (Gaulis, 2001 : 131, 240), dans le sillage des pièces du montreur d'ombres Dimitris Katsoulis, publiées à Melbourne en 1983 (Kanarakis, 1985 : 529-533). Vasso Kalamara, longtemps éloignée du monde anglophone, fait revivre les genres traditionnels de la littérature orale grecque en en transposant les personnages et les thématiques en Australie. Enseignante de grec moderne, elle entend jouer un rôle de passeur culturel entre les deux pays, ou plutôt de la Grèce à l'Australie. Elle déclare ainsi avoir traduit en anglais avec David Hutchison ses propres pièces sur les aventures de Karaghiozis en Australie, en vue de faire connaître l'univers du théâtre d'ombres grec au public anglophone ${ }^{3}$.

Présenté par l'auteur comme un tournant, le recueil Genèse (Genesis, 1993), écrit en anglais, dont les poèmes sont cette fois qualifiés de " poèmes australiens » («Australian poems »), invite peut-être à réexaminer l'évolution du regard de l'auteur sur cette problématique, notamment après la publication annoncée de son prochain recueil de nouvelles, Les Expatriés ${ }^{4}$. Pour l'heure, les Grecs en Australie sont toutefois toujours chez Vasso Kalamara des exilés, des étrangers dans le pays d'accueil, sans possibilité de retour au pays natal; leur mort est conçue comme une seconde mort, après la première mort que constitue l'exil, suivant le motif de la chanson traditionnelle. Ainsi, le personnage de Nelly dans Impressions d'un voyage (Endypossis apo ena taxidi, 1977) tente de rentrer en Grèce, mais son retour au pays est un échec qui la contraint à reprendre le bateau pour l'Australie, pour certainement y mourir comme les personnes âgées de la nouvelle "Les retraités » («I syndaxiouchi ») du recueil Autres terres. Le titre du premier livre en prose de l'auteur, Autres terres, suggère du reste bien que l'Australie, irréductiblement marquée par une altérité hostile, ne permet pas aux immigrés, presque tous grecs dans son œuvre, de se construire une vie et une identité.

Les nouvelles d'Antigone Kefala, écrites en anglais, proposent une toute autre approche. Les personnages principaux y sont en effet souvent des immigrés non plus de première génération, comme chez Vasso Kalamara, mais de deuxième génération, qui sont certes considérés comme étrangers dans la société urbaine dans laquelle ils vivent, mais intégrés à celle-ci. Les narratrices, où l'on reconnaît beaucoup de traits de l'auteur elle-même, sont des personnages en quête, dans

3. $C f$. la notice biographique en grec de l'édition grecque de sa pièce Olympias, mère d' Alexandre le Grand (Kalamara, 2001).

4. La directrice des éditions Owl Publishing, Helen Nickas, nous précise par courrier au début de l'année 2008 que la date de publication de ce recueil n'est pas encore connue. 
des récits menés à la première personne, et non à la troisième personne comme chez Vasso Kalamara. Les immigrés de première génération, s'ils exercent une fascination et constituent un repère moral et esthétique, comme la tante Niki dans L'Île (The Island, 1984), sont cependant toujours des personnages secondaires. Les personnages principaux, immigrés tous grecs, sont dans une situation d'entre-deux qui, dans la prose d'Antigone Kefala, s'avère tantôt ludique tantôt dramatique. Dans son récit Alexia (1984), qualifié de conte de deux cultures ( a tale of two cultures ») dans la première édition (Kefala, 1984), puis de conte pour grands enfants ( a tale for advanced children ») dans la réédition bilingue de 1995 (Kefala, 1995), les mots étrangers, que l'enfant s'approprie, sont l'objet de rêverie poétique, tandis que la narratrice de L'Ille observe l'incongruité des mots de sa langue maternelle dans ce nouveau pays :

« L'autre soir, j'entendais madame Koussis, sa voix montant et descendant sur ces expressions de miel [...] ma poupée [...] mon trésor [...] qui venaient si facilement, si naturellement en grec, sans ostentation, comme une ancienne et discrète litanie avec laquelle on a grandi. Maintenant elle semblait les utiliser de façon creuse, sans enthousiasme, une vieille habitude. » (Kefala, 2002 : 67, trad. de Marie Gaulis)

Pourtant, la narratrice cherche à se trouver une place dans le pays d'accueil, ce que l'écrivain rend de façon intéressante : le nom de la narratrice, Mélina, n'est donné que dans la deuxième partie du roman, au théâtre, au moment de l'entrée en scène du personnage (Kefala, 2002:88-89). Le premier livre d'Antigone Kefala s'intitule du reste L'Étrangère (The Alien, 1973) : ce n'est plus le pays d'accueil qui est conçu dans son altérité, comme dans Autres terres de Vasso Kalamara, mais le sujet immigré. Et alors que Vasso Kalamara enseigne le grec moderne aux Australiens, Antigone Kefala enseigne l'anglais langue étrangère aux immigrés ${ }^{5}$.

En outre, si les personnages de Vasso Kalamara sont porteurs d'une identité grecque, ceux d'Antigone Kefala se sentent certes Grecs, mais plus généralement Européens, ce qui n'est pas étonnant quand on songe que l'auteur est issue de la diaspora grecque de Roumanie. Leur culture de référence est la culture européenne classique, en particulier française - on trouve, dans la prose d'Antigone Kefala, des références fréquentes à Racine, cité en français dans L'Île, ou à Baudelaire (Kefala, 2002 : 100-101). La Grèce, c'est-à-dire la Grèce helladique, est, comme souvent chez les auteurs de la diaspora, une Grèce image, liée à l'esthétique particulière du paysage, qui est par exemple mise à distance par la photographie dans L'Île :

« Nos vies passées dans les bibliothèques, tous penchés sur les mêmes tables, comme si ces derniers mois nous n'avions pas bougé de là. J'essayais de me réconforter en regardant un livre avec des photographies de Grèce que Kate avait découvert. Voilà que, page après page, tout y était, fixé pour toujours, la sensation délicate et transparente du paysage, le soleil qui ruisselait de blancheur sur l'architecture délabrée des maisons et des rues, le poids sombre des gens, les femmes penchées, immobiles, sur les champs. " (Kefala, 2002 : 154-155, trad. de Marie Gaulis)

Et, alors que chez Vasso Kalamara les Aborigènes sont considérés comme autres, chez Antigone Kefala les peuples premiers des antipodes

« nous ressembl[ent], à nous et à ceux de la colonie étriquée au sein de laquelle nous paraiss[ons] nous mouvoir, tous des déracinés qui parl[ent] constamment du passé. » (Kefala, 2002 : 45, trad. de Marie Gaulis)

Il est enfin intéressant de noter qu'Antigone Kefala ne mentionne avec précision le lieu dans ses récits que lorsque l'action se déroule en Australie : quoique aisément reconnaissables, Bucarest dans Le Premier Voyage (The First Journey, 1975) et Wellington dans L'Île et dans Alexia ne sont pas nommées, tandis que Sydney l'est explicitement dans La Pension (The Boarding House, 1975). C'est l'Australie qui apparaît comme propice au lent épanouissement des personnages d'Antigone Kefala, qui sont en quête d'une mesure, pour reprendre les premiers vers du poème éponyme $\mathrm{du}$ recueil Temps assoiffé (Thirsty Weather, 1978) :

« Trouver notre mesure, exactement, non pas l'écho d'autres voix. » (Kefala, 2000 : 24)

Ces vers pourraient constituer la devise des narrateurs de Christos Tsiolkas. Avec cet écrivain né en Australie, Grec de deuxième génération qui écrit exclusivement en anglais, l'approche de la problématique identitaire change une nouvelle fois. Son premier livre, Chargé (Loaded, 1995), premier roman de notre corpus, est construit en quatre parties d'inégale longueur, dont chacune porte pour titre l'un des points cardinaux, correspondant aux différentes banlieues de Melbourne que le narrateur arpente en quête de plaisirs fugitifs. Dans cette narration menée à la première personne, largement autobiogra- 
phique, écrite « avec colère » ${ }^{6}$, comme le précise l'auteur dans la préface à l'édition grecque (Tsiolkas, 1999a: 8), on peut lire un retournement de la problématique identitaire :

« Je ne suis pas australien, je ne suis pas grec, je ne suis rien. Je ne suis pas un ouvrier, je ne suis pas un étudiant, je ne suis pas un artiste, je ne suis pas un junkie, je ne suis pas causeur, je ne suis pas un Australien, pas un wog, rien. Je ne suis pas de gauche, pas de droite, du centre, de la gauche du centre, de la droite de Genghis Khan. Je ne vote pas, je ne manifeste pas, je ne fais pas la charité. Un fugitif, voilà ce que je suis. » (Tsiolkas, 1997 : 149)

Ces négations, prises dans la figure circulaire des rythmes ternaires, ne vont pas sans se contredire: "Je ne suis pas blanc, je suis un wog » (Tsiolkas, $1997: 5$ ). Wog est en outre le premier mot que Christos Tsiolkas et Sasha Soldatow font figurer sur la carte de leur ville à la fin du chapitre «Esthétique» («Aesthetics») de leur autobiographie Jump Cuts (1996) (Soldatow and Tsiolkas, 1996 : 293). Insulte proférée à l'encontre des immigrés originaires d'Europe du Sud ou de Méditerranée orientale, le terme $\operatorname{wog}^{7}$ est aujourd'hui revendiqué par les enfants de ces immigrés, comme le montrent, outre l'œuvre de Christos Tsiolkas, les spectacles et les films interprétés par l'acteur Nick Giannopoulos, comme le long métrage The Wog Boy (2000) d'Aleksi Vellis, sur un mode humoristique cette fois. Élevés dans une culture mixte, ces artistes revendiquent une culture wog, partie intégrante de l'identité australienne contemporaine, et s'autorisent à critiquer tant le pays et la société d'accueil que le pays et la société d'origine, comme le fait Christos Tsiolkas dans ses romans L'Homme de Jésus (The Jesus Man, 1999) et L'Europe morte (Dead Europe, 2005) (Tsiolkas, 1999 b et 2005). L'auteur confie par exemple avoir été choqué, lors d'un séjour en Grèce, par le nationalisme ambiant et par l'intolérance des Grecs à l'égard des nouveaux immigrés, la Grèce, traditionnellement pays d'émigration, étant devenue dans les années 1990 terre d'immigration. Il écrit :

«Ivre un soir dans une taverne à Athènes, j'ai entendu une bande de Grecs à la table voisine insulter les Albanais ; je me suis levé et j'ai déclaré à la tablée "Moi aussi je suis albanais". Je voulais dire que moi aussi je suis un immigré. » (Tsiolkas, 1999a : 8)

C'est une double culture qui se manifeste, parfois avec rage, dans l'œuvre de Christos Tsiolkas: le narrateur de Chargé, à la fin de chaque face de sa cassette préférée, a par exemple recopié, à la suite de chansons anglosaxonnes, une célèbre chanson grecque (Tsiolkas, 1997 : 24). Dans la préface à l'édition grecque de son roman, l'auteur résume sa position à l'égard de la question identitaire de manière assez poétique :

« En fin de compte, je ne suis ni grec ni australien. Je vis dans le trait d'union qui lie ses deux mots. » (Tsiolkas, 1999a : 9)

Notons enfin que le genre du roman, privilégié par Christos Tsiolkas, contrairement à Vasso Kalamara et Antigone Kefala, qui préfèrent la nouvelle (short story ou diiyima pour la première, novella ou nouvela pour la seconde), ne relève peut-être pas d'un choix anodin. En revendiquant une prise de possession du territoire et même des territoires pluriels de l'identité grécoaustralienne, l'auteur entend construire et dire le monde dans sa totalité plutôt que de travailler sur l'ellipse, le non-dit, le silence et l'absence, comme le permet si bien le genre de la nouvelle ; et le titre de son premier livre, Chargé, qui se prête certes à de multiples interprétations, suggère en tout cas un trop plein, à la différence des textes des prosateurs gréco-australiens des générations précédentes.

\section{De l'auteur au lecteur}

Les différences d'approche de l'identité grécoaustralienne qui apparaissent chez ces trois auteurs ne sont pas sans effet sur la diffusion et sur la réception des œuvres. Les travaux sur le multiculturalisme ont à notre sens tendance à surestimer le critère de la langue dans l'évaluation de la diffusion de la littérature des immigrés de première ou de deuxième génération. En effet, la traduction, largement pratiquée en Grèce et bien subventionnée en Australie, permet de pallier l'obstacle de la langue étrangère ; elle permet même, dans le cas de l'édition bilingue, de mieux apprécier le texte original. Pourtant, les trois auteurs de notre corpus semblent cantonnés à des lectorats très différents.

Les premiers livres de Vasso Kalamara, Gouttes (Stalagmaties, 1959), Autres terres (1961), Amertumes (Pikres, 1976), Impressions d'un voyage (1977) et Le Fermier et le sculpteur (O mandrakas kai o glyptis, 1979), paraissent à Athènes, mais, publiés à compte d'auteur, ils ne

6. Nous traduisons à partir de la traduction grecque. L'original n'a à notre connaissance pas été publié.

7. Les dictionnaires proposent plusieurs étymologies pour ce terme que l'on trouve en anglais britannique et australien : il est tantôt l'acronyme de « Worthy Occidental Gentleman », tantôt l'abréviation de « golliwog». 
connaissent qu'une diffusion confidentielle en Grèce. Seul son recueil de nouvelles, Autres terres, est réédité en 1980 par la grande maison d'édition athénienne Estia, mais cette publication reste sans suite. La thématique de ces textes permet en revanche à l'auteur de tirer profit de la politique favorable au multiculturalisme, à l'œuvre en Australie depuis les années 1970, pour faire publier ces textes, avec des traductions en anglais assurées par ses propres étudiants, comme Reg Durack ou June Kingdom. Si les recueils de poésie Vingt-deux poèmes et Paysage et âme paraissent en édition bilingue à Perth à compte d'auteur en 1977 et en 1980, nombre de ses textes sont publiés par des maisons d'édition à Perth, Melbourne ou Fremantle, qui souvent bénéficient de subventions étatiques : les recueils de nouvelles Autres terres et Amertumes sont réédités en bilingue en 1977 et en 1983, la pièce de théâtre Une souricière à pain (Mia faka me psomaki, 1986) est éditée en bilingue, le recueil de nouvelles La Même Lumière (The Same Light, 1989), constitué de textes déjà parus ou inédits, est publié en traduction anglaise, la pièce de théâtre Olympias (2001) paraît simultanément en grec et en anglais.

Antigone Kefala est, quant à elle, reconnue en Australie ${ }^{8}$, même si ses textes sont surtout publiés par de petites maisons d'édition (comme Wild and Woolley, Hale and Iremonger, John Ferguson à Sydney). Elle est en revanche quasiment inconnue en Grèce. Plusieurs de ses œuvres sont pourtant traduites en grec par l'universitaire et éditrice Helen Nickas ${ }^{9}$, mais ces traductions sont publiées à Melbourne, chez Owl Publishing, petite maison d'édition spécialisée dans la littérature de la diaspora grecque : Alexia (1984) est ainsi réédité en 1995 en édition bilingue anglais-grec, un choix de poèmes extraits des recueils L'Étrangère (1973), Temps assoiffé (1978), Carnet européen (European Notebook, 1988) et Absence (Absence, 1992), est publié sous le titre Poèmes (Poems) en 2000, avec une traduction grecque en regard, et la nouvelle L'île est rééditée en 2002 en édition trilingue anglaisfrançais-grec. Ces rééditions où deux ou trois des langues de cet auteur quadrilingue sont présentes témoignent de la volonté, partagée par l'auteur et l'éditrice ${ }^{10}$, de mettre en avant la culture cosmopolite et plurilingue du monde grec de la diaspora. En outre, les illustrations de Nikos Kypraios pour Alexia et des artistes lituano-australiens Jurgis et Jolanta Janavicius pour Poèmes et L'Île inscrivent ses rééditions dans le champ de l'Australie multiculturelle: Jurgis Janavicius déclare par exemple à cette occasion chercher à représenter les spécificités de l'arrière-pays australien dans sa nudité, proche selon lui de la poésie d'Antigone Kefala (Kefala, $2000: 116$ ).

Les romans de Christos Tsiolkas, enfin, sont publiés par Random House, éditeur qui jouit d'une large diffusion tant en Australie que dans le reste du monde anglophone. Ils font déjà l'objet de longs développements dans certaines histoires de la littérature australienne (Vernay, 2008 : 221), et certains d'entre eux sont traduits en allemand (Chargé), en grec (Chargé et L'Homme de Jésus) ou en turc (L'Europe morte). Les traductions grecques de Chargé et de L'Homme de Jésus sont publiées en 1999 et en 2001 par un petit éditeur athénien, Oxy, spécialisé dans la littérature alternative (Tsiolkas, 1999a et 2001), à la suite du succès du film d'Ana Kokkinos, Head on (1998), adaptation de Chargé ${ }^{11}$. L'édition grecque de Chargé montre que c'est davantage la thématique sexuelle, en l'occurrence gay, qui retient l'attention à Athènes plutôt que la complexité d'une identité wog. L'édition française du DVD du film d'Ana Kokkinos, dans la collection "Cinégay » chez Antiprod, procède du reste d'une démarche analogue: ainsi, dans le synopsis que propose la jaquette du DVD, il n'est par exemple pas précisé que le personnage principal, Ari, interprété par Alex Dimitriades, est fils d'immigrés.

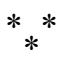

D'une littérature de l'exil (avec Vasso Kalamara) à une culture wog revendiquée (avec Christos Tsiolkas), l'étude de ces trois prosateurs gréco-australiens contemporains permet de suivre l'évolution complexe des problématiques identitaires dans l'Australie multiculturelle, qui a des effets, au plan littéraire, sur le genre (de la nouvelle au roman), sur les techniques d'écriture (de l'usage de la troisième personne à celui de la première personne dans la conduite du récit) et

8. Marie Gaulis rapporte les propos du critique Geoff Page qui affirme qu'Antigone Kefala est «l'un des premiers poètes d'origine non anglophone à avoir eu un impact sur la poésie australienne contemporaine et l'un des meilleurs » (Gaulis, 2001 : 183).

9. Qu'il me soit ici permis de remercier Helen Nickas qui m'a envoyé de Melbourne nombre de textes introuvables en Europe.

10. Cf. la partie « Why a trilingual text and who is it for? » dans l'introduction de Helen Nickas à la réédition de L'Île (Kefala, $2002: 22-23)$.

11. Le titre Kata metopo [Frontalement] (Tsiolkas, 1999a), choisi pour l'édition grecque du roman, est du reste la traduction du titre de cette adaptation cinématographique. 
sur la thématique (du Grec perdu dans une Australie rurale hostile au wog urbain, fugitif et gay). En fonction des approches retenues par les auteurs et de la gestion politique du multiculturalisme, ces textes, regroupés sous la catégorie « littérature gréco-australienne », bénéficient en fait d'une diffusion très diverse auprès du lectorat tant anglophone que hellénophone, en Grèce comme en Australie, et s'inscrivent dans des champs littéraires différents.

L'œuvre de ces auteurs est en outre l'occasion d'évoquer des aspects essentiels de la culture australienne qui, jusqu'à maintenant, n'ont guère retenu l'attention des chercheurs européens. Pourtant, la revendication nouvelle de cette identité wog apparaît comme l'un des objets d'étude les plus intéressants dans la société australienne contemporaine, phénomène certes loin des mythes exotiques d'une Australie qui invite au rêve, mais proche des réalités d'un continent viscéralement lié au nôtre.

\section{BIBLIOGRAPHIE}

Gaulis Marie, 2001. Une littérature de l'exil: Vasso Kalamara et Antigone Kefala, deux écrivains grecs d'Australie, Genève, Slatkine, Travaux des universités suisses 7 .

Harel Simon, 2005. Les Passages obligés de l'écriture migrante, Montréal, XYZ, Théorie et littérature.

Kalamaras Vasso, 1977. Other Earth, trad. de Reg Furack et de l'auteur, Fremantle, Fremantle Arts Centre Press.

- [Kalamara Vasso-Lone], 1980a. Alla chomata [Autres terres], Athènes, Estia, Littérature néogrecque 237.

_, 1980b. Landscape and Soul, trad. de June Kingdom et de l'auteur, Perth, s.e.

- [Kalamara Vasso], 2001. Olympias, mitera tou Megalou Alexandrou [Olympias, mère d'Alexandre le Grand], Melbourne, Owl Publishing, Writing the Greek Diaspora.

KANARAKIs Yorgos, 1985. I logotechniki paroussia ton Ellinon stin Afstralia [La Présence littéraire des Grecs en Australie], Athènes, Fondation des Études néo-helléniques, Études 1.
- [KanaraKis George], 1987. Greek Voices in Australia: A Tradition of Prose, Poetry and Drama, Sydney, Australian University Press.

Kefala Antigone, 1984. Alexia. A Tale of Two Cultures, Sydney, John Ferguson.

—, 1995. Alexia. A Tale for Advanced Children, traduit en grec et préfacé par Helen Nickas, Melbourne, Owl Publishing, Writing the Greek Diaspora.

—, 2000. Poems, traduit en grec et préfacé par Helen Nickas, Melbourne, Owl Publishing, Writing the Greek Diaspora.

_, 2002. The Island, préface de Helen Nickas, traduit en grec par Helen Nickas et en français par Marie Gaulis, Melbourne, Owl Publishing, Writing the Greek Diaspora.

NicKas Helen, 1992. Migrant Daughters. The Female Voice in Greek-Australian Prose Fiction, Melbourne, Owl Publishing.

Pons Xavier, 1996. Le Multiculturalisme en Australie. Au-delà de Babel, Paris, L'Harmattan.

SAwAs Stéphane, 2007. Des écritures migrantes à une littérature de diaspora : l'exemple des écrivains émigrés grecs, communication au colloque La Migrance à l'œuvre : repérages éthiques, esthétiques et politiques, Dublin, University College Dublin.

_, 2008. Les expressions littéraires des diasporas grecques : une marge de la littérature néo-hellénique ?, in Corinne Alexandre-Garner (éd.), Frontières, marges et confins, Nanterre, Presses universitaires de Paris X, Chemins croisés, pp. 51-63.

Soldatow Sasha and Christos Tsiolkas, 1996. Jump Cuts, Milsons Point, Random House.

Tsiolkas Christos, 1997. Loaded, Londres, Random House.

_, 1999a. Kata metopo [Frontalement], trad. de Yannis Polyzos, Athènes, Oxy, Dé-formation.

-, 1999b. The Jesus Man, Milsons Point, Random House.

—, 2001. O anthropos tou Iissou [L'Homme de Jésus], trad. de Nérina Kiosséoglou, Athènes, Oxy, Dé-formation.

-, 2005. Dead Europe, Milsons Point, Random House.

Vernay Jean-François, 2008. Panorama du roman australien des origines à nos jours, 1831-2007, Paris, Hermann, Savoir Lettres.

WAPSHERE Nathaniel, 1994. Le multiculturalisme australien, Hommes \& Migrations 1774, pp. 6-13. 
